\title{
Gestión de costos en el sector de áridos a través del método de costeo basado en actividades*
}

\author{
Aggregates Industry Cost Management Through the Methodology of Activity Based Costing \\ Gestão de custos no setor de agregados através do método de Custo Baseado em Atividades
}

\author{
Cecilia del Pilar Gallegos Muñoz ${ }^{\mathrm{a}}$ \\ Universidad del Bio-Bio, Chile \\ cecilia@ubiobio.cl \\ ORCID: http://orcid.org/0000-0003-0846-2173 \\ Estela Irene Rodríguez Quezada \\ Universidad del Bio-Bio, Chile \\ ORCID: http://orcid.org/0000-0002-3259-0936
}

DOI: https://doi.org/10.11144/Javeriana.cc21.gcsa

Recepción: 25/04/2019

Aceptación: 21/10/2019

Publicación: 05/06/2020

\section{Resumen:}

El presente estudio tiene como objetivo identificar oportunidades de mejora y reducción de costos en una empresa productora de áridos, a través de la metodología de Costo Basado en Actividades ( $\mathrm{ABC}$ ). El estudio de caso se realiza con visitas a terreno; entrevistas al dueño, gerentes y encargados de los procesos; y revisión de informes financieros y contables. Los resultados muestran como el $\mathrm{ABC}$ permite conocer el costo y rentabilidad de cada uno de los productos de la empresa, medido en metros cúbicos. Además, está metodología permite identificar las actividades que significan un mayor costo y aquellas que no agregan valor a la compañía. De este modo, es posible prever oportunidades de mejora de procesos que puedan conducir a una reducción de costos en el futuro.

Código JEL: M20, M41, O14

Palabras clave: gestión de costos, costeo basado en actividades, producción de áridos, costos indirectos.

\section{Abstract:}

This study aims to identify opportunities for improvement and cost reduction in a company that produces aggregates through the methodology of Activity Based Costing (ABC). This case study is conducted with field visits; interviews with the owner, managers and process managers; and reviews of financial and accounting reports. The result shows how the $\mathrm{ABC}$ allows knowing the cost and profitability of each one of the company products, measured in cubic meters. Furthermore, this methodology enables to identify the activities that represent a higher cost and those that do not add value to the company. In this way, it is possible to foresee opportunities for improvement that might lead to significant cost reductions in the future.

JEL Code: M20, M41, O14

Keywords: Cost management, activity-based costing, aggregate production, indirect costs.

\section{Resumo:}

O presente estudo tem como objetivo identificar oportunidades de melhoramento e redução de custos em uma empresa produtora de agregados, através da metodologia de Custo Baseado em Atividades (ABC). O estudo de caso realiza-se com visitas a terreno; entrevistas ao dono, gerentes e pessoas encarregadas dos processos; e revisões de informes financeiros e contábeis. Os resultados mostram como o ABC permite conhecer o custo e a rentabilidade de cada um dos produtos da empresa, medido em metros cúbicos. Além disso, esta metodologia permite identificar as atividades que significam um maior custo e aquelas que não acrescentam valor à companhia. Deste modo, é possível prever oportunidades de melhora de processos que possam conduzir a uma redução de custos no futuro.

Código JEL: M20, M41, O14

Palavras-chave: gestão de custos, custeio baseado em atividades, produção agregada, custos indiretos.

Notas de autor

a Autora para correspondencia. Correo electrónico: cecilia@ubiobio.cl. 


\section{Introducción}

El método de costeo basado en actividades - $\mathrm{ABC}$ - es una herramienta de gestión y medición de costos que permite a las empresas obtener costos más precisos, así como detectar capacidades no utilizadas o actividades que no agregan valor a la compañía. Esto se traduce finalmente en una reducción de sus costos y un aumento de las utilidades.

El ABC surge a partir de la década de 1980, sin embargo, de acuerdo a la literatura vigente, no se encuentra evidencia de su aplicación en pequeñas y medianas empresas en Chile. Es común observar que en este tipo de empresas no son utilizados sistemas formales, dado que sólo acumulan sus costos y gastos indistintamente, y los distribuyen entre sus productos o servicios de manera proporcional al volumen de producción. Otras, en cambio, implementan sistemas de costos tradicionales, obteniendo información poco fiable y determinando costos poco precisos, lo que impide a su vez que puedan tomar buenas decisiones (Artieda, 2015).

Sin embargo, las micro y pequeñas empresas requieren implementar un sistema de gestión como un elemento básico que permita la determinación de costos de producción de bienes y servicios para la toma de decisiones, así como establecer el precio de venta para obtener una ventaja comparativa. En este contexto, una de las mejores herramientas para el mejoramiento de un sistema de costeo, inclusive por parte de pequeñas empresas es el ABC (Arellano Quispe, Ayaviri y Escobar, 2017, p. 40).

En la literatura se encuentran diversas metodologías para obtener el costo de un producto, por lo que se debe elegir aquella que proporcione información adecuada para elaborar estrategias a fin de cumplir objetivos, calcular correctamente la rentabilidad y alcanzar una posición competitiva (Ríos, Muñoz y Rodríguez, 2014, p. 2). Cada sistema de costos tiene sus características y puede tener diferentes resultados, por lo tanto, la elección debe corresponder al propósito y objetivos de la empresa (Artieda, 2015; Baldini y Casari, 2008).

El ABC consiste fundamentalmente en asignar costos a todas las actividades de la empresa identificadas como relevantes, a través de inductores y, posteriormente, asignar el costo de dichas actividades a los objetos de costo por medio de inductores (Toro, 2016).

Este artículo analiza un estudio de caso de una empresa dedicada a la actividad económica de la producción de áridos. Se denominan productos áridos a las partículas granulares de material pétreo de tamaño variable que se originan por fragmentación de las distintas rocas de la corteza terrestre, en el cual actúan procesos de selección o chancado para obtención de distintos productos.

Estos procesos requieren mano de obra y maquinarias, lo cual genera mayoritariamente costos indirectos, lo que justifica la aplicación del método $\mathrm{ABC}$ para calcular sus costos. De este modo, podrían obtenerse datos más precisos que aquellos obtenidos a través de métodos tradicionales. Por lo tanto, el objetivo de este artículo es identificar oportunidades de mejora y reducción de costos en una empresa productora de áridos a través de la metodología de costo basado en actividades.

\section{Marco teórico}

El sistema ABC fue propuesto por primera vez por Kaplan y Cooper en la década de 1980. En ese entonces la mano de obra directa y los materiales eran los factores determinantes de la producción y los costos indirectos no representaban una gran proporción del costo total. Sin embargo, con el tiempo, los sistemas tradicionales comenzaron a presentar falencias, dado que la proporción de costos indirectos se vio incrementada debido al aumento en el uso de las tecnologías. Fue a raíz de esas falecias en el cálculo de los costos indirectos que surgió el sistema de costeo basado en actividades.

Brito, Ferreiro y López (2009) define el ABC como "un proceso gerencial que ayuda en la administración de actividades y procesos del negocio, durante la toma de decisiones estratégicas y operacionales" (Brito et al., 2009 , p. 9). Por tanto, el método ABC es una herramienta que muestra una mejor visualización de los costos 
por medio del análisis de las actividades ejecutadas dentro de la organización y sus relaciones con los productos o servicios (Vieira et al., 2016). Para Lima y Júnior (2017), el método ABC se trata de una herramienta de análisis estratégico más amplia, pues, posibilita el surgimiento de oportunidades de optimización de retornos estratégicos y una mejor gestión sobre el consumo de los recursos.

Para la correcta implementación del sistema de costeo basado en actividades se debe contar con información exhaustiva relacionada con la producción y los costos, sobre una base sistemática y oportuna. Esta información se obtiene de su sistema de contabilidad, siempre y cuando se haya sistematizado y estandarizado en forma apropiada todos los factores de producción (Coronel, 2014, p. 18).

La revisión de la literatura disponible permite identificar principalmente dos metodologías para desarrollar el costeo ABC: la metodología de las dos fases y la de los cuatro pasos. La primera de ellas, contiene fases y etapas. En una primera fase se determinan los costos de las actividades de cada centro de costo, considerando seis etapas. En su segunda fase, la cual está compuesta de dos etapas, se determinan los costos de los productos (Sanhueza, 2015).

Por su parte, la metodología de los cuatro pasos considera desarrollar un diccionario de actividades, determinar el gasto que involucra cada actividad; identificar productos, servicios y clientes; y diseñar generadores de costos que relacionen actividades con productos o servicios (Torres, Salete y Delgado, 2017, p. 647).

La metodología del $\mathrm{ABC}$ se sustenta, por lo tanto, en la actividad como base conceptual, dado que las actividades son las que ocasionan los costos y de que los productos o servicios consumen actividades. Este sistema asigna costos a las actividades a través de inductores de costos de recursos, basándose en cómo estos son consumidos. Posteriormente se asignan las actividades a los objetos de costo por medio de nuevos inductores (Brito et al., 2009).

En este sentido, es fundamental centrarse en las actividades que realiza la empresa con una visión horizontal y siguiendo el flujo de los procesos. Con esto, es posible descomponer los costos de los productos en las actividades, permitiendo analizar en forma más realista y profunda, las posibilidades de reducción de costos (Brito et al., 2009). Igualmente, es importante entender una actividad como el resultado de la combinación de recursos humanos, materiales, tecnológicos y financieros utilizados en la producción de bienes y servicios de un negocio (Martins, 2008).

Por su parte, los recursos son definidos como cualquier elemento económico aplicado o utilizado en el desarrollo de una actividad. El costo de los recursos es asignado a las actividades a través de direccionadores de costos. Los direccionadores de costos o inductores son la base del ABC y tratan de rastrear el orginen del costo y establecer una relación de causa efecto. Martín (2017) define como direccionador o cost-driver ${ }^{1}$, a la unidad cuantitativa que mide los consumos de recursos necesarios para realizar las actividades. Estos puede sen clasificados como inductores de transacción cuando todos los resultados requieren las mismas demandas de actividades; inductores de duración cuando miden la cantidad de tiempo necesario para realizar una actividad; e inductores de intensidad cuando miden el uso directo de recursos que una actividad requiere cada vez que se realiza (Rincón, 2011).

La elección de un inductor correcto requiere comprender la relación entre recursos, actividades y objetos de costos (López, Gómez y Marín, 2011, p. 7). Se debe elegir aquel que sea más representativo de la relación causa efecto y que sea más fácil de medir y observar. La cantidad de cada direccionador que está asociada a la actividad que se quiere costear se denomina factor de consumo de recursos o inductor de recursos (Medianeira, 2011). La cantidad de direccionadores con que se trabajará dependerá del nivel de precisión que se desea obtener y la relación costo beneficio deseado. Los direccionadores pueden variar de una empresa a otra, pues dependen de cómo y por qué se ejecutan las actividades (Santos y Padrones, 2015).

$\mathrm{Al}$ revisar la literatura relacionada a la contabilidad de gestión, los costos $\mathrm{ABC}$ constituyen los tópicos de mayor interés por los investigadores, sumando un $43 \%$ de las investigaciones publicadas en la materia. Entre los tópicos investigados de este método de costeo, su aplicación específica en diferentes sectores 
industriales acapara más del 20\% de la literatura existente (Bustamante, 2015). En este sentido, el ABC es una de las líneas de investigación más importantes de la contabilidad de gestión. Se encuentra en la literatura trabajos que estudian los antecedentes y la fundamentación, las potencialidades, las limitaciones y los factores que inciden en la implementación de este sistema. También se encuentran algunas evidencias que documentan la aplicación en sectores específicos, especialmente en organizaciones de servicios, salud, energía y en empresas manufactureras del sector privado de países desarrollados (Balmaceda y Guerra, 2017; Cataldo, 2008; Cárdenas, 2011; Molina y Reyes, 2011; Raupp y Beuren, 2011). Sin embargo, es notable el deficit de trabajos empíricos que den cuenta de la implementación del método $\mathrm{ABC}$ en países en desarrollo (Fei y Isa, 2010).

Entre las ventajas del sistema de costo $\mathrm{ABC}$ se encuentran: lograr un mejor control y reducción de los costos indirectos, debido a que permite la identificación y posterior eliminación de actividades que no agregan valor. Además, se pueden calcular de forma más precisa los costos, lo cual es muy útil en la etapa de planeación, pues suministra información que sirve de guía para varias decisiones. Con el $\mathrm{ABC}$ se puede obtener información valiosa para decisiones como la fijación de precios, la introducción de nuevos productos y la adopción de nuevos diseños o procesos de fabricación (Brito et al., 2009, Reinheimer et al., 2010). De esta forma, es posible tomar decisiones estratégicas acertadas y analizar cómo se encuentra el precio de venta en relación con la competencia (Cuevas, Chávez, Castillo, Marino y Solarte, 2004). La información generada por el ABC permite reestructurar los procesos de negocios al detectar productos que no generan valor, incrementar la rentabilidad, así como tomar mejores decisiones en la subcontratación, aceptación de pedidos, combinación de ventas y el desarrollo de nuevos productos (Gómez, 2014; Ríos, Rodríguez y Ferrer, 2012). Por su parte, Morillo y Cardozo, (2017) señalan que además el ABC permite reorganizar, listar y conceptualizar cada actividad desarrollada en las distintas áreas de la organización.

Encuestas internacionales en países desarrollados muestran la existencia de una alta percepción de utilidad de los sistemas ABC (Bhimani et al., 2007). El estudio de Strasorier (2012) destaca la disponibilidad de datos e información resultante, no solamente referidos a los costos sino también a otros aspectos inherentes al desarrollo de la organización. De este modo se contribuye a facilitar las tareas de control y supervisión de dichos procesos. Otras de las ventajas atribuibles al sistema $\mathrm{ABC}$ es la posibilidad de reducir líneas de productos, determinar actividades no rentables, reorganizar operaciones y ampliar nuevos mercados, ya que es un modelo gerencial y no solo contable (Sánchez, 2013, p. 72).

Sin embargo, la implementación del $\mathrm{ABC}$ presenta algunos inconvenientes que enumeramos a continuación: 1) La falta de recursos que pudieran tener las empresas para la implementación y la contratación de consultorías (Brito et al., 2009; Prieto, Santidrián y Valladares, 2007); 2) La resistencia al cambio y necesidad de capacitación y actualización de conocimientos por parte de contadores y directivos (López et al., 2011; Prieto y Valladares, 2013); 3) La inoperatividad del sistema cuando se trabaja con un número muy alto de inductores, ya que la selección y construcción de ellos es un proceso complejo que requiere un amplio conocimiento de la metodología y organización, así como de sistemas de información que faciliten la recolección y análisis de los datos (Brito et al., 2009; Prieto et al., 2007); 4) La resistencia que puede provocar el que su implementación se realice de forma aislada o independiente en departamentos de contabilidad o informática, sin incluir a toda la organización (Prieto y Valladares, 2013, p. 21).

Respecto a las motivaciones de las empresas para implementar el ABC, Ríos et al. (2014) señalan que estas motivaciones pueden variar de una empresa a otra y pueden, al contrario de lo que podría pensarse, no estar relacionadas con la reducción de costos. Muchas veces, las razones tienen que ver con la mejora de la información de costos de bienes y servicios, o la necesidad de revisar su política de precios debido a un aumento en la cantidad de competidores, entre otras. Sin embargo, independiente de la razón que tenga cada empresa es importante que exista una definición previa del propósito de dicha implementación y la información precisa que se quiere obtener con este sistema, con el fin de obtener éxito en la implementación, sobre todo en las pequeñas empresas. Cuando se ha definido claramente el fin que persigue la implementación 
del $\mathrm{ABC}$ es posible romper con una de las principales barreras en este tipo de empresas, que es la excesiva complejidad de diseño y funcionamiento. Una gran cantidad de actividades implica la creación de demasiados inductores, lo que termina incrementando la dificultad del sistema, arruinando su funcionamiento (Morillo y Cardozo, 2017, p. 105).

Finalmente, es importante señalar que al momento de implementar el $\mathrm{ABC}$, es recomendable que en un primer momento éste se base en datos reales, objetivos y documentados, generados por el mismo sistema contable tradicional de la empresa (Chea, 2011). Respecto a la definición del número de actividades, existen diversas teorías sobre cuánto es el número apropiado. Por su parte, Kaplan y Cooper (2003) sugieren que para tomar este sistema de manera simple debe incluir alrededor de 30 a 50 actividades. Sin embargo, Ríos et al. (2014, p. 230) señalan que la mayoría de las empresas definen sus actividades por el centro de responsabilidades o los departamentos que existen.

\section{Metodología}

Esta investigación es de tipo cualitativa y participativa, basada en un estudio de caso, aplicada, semiexperimental e histórica. Tiene como unidad de investigación a una empresa productora de áridos ubicada en la ciudad de Chillán, Chile.

Para el trabajo de campo se utilizaron instrumentos como la revisión documental, la observación en terreno y entrevistas semiestructuradas aplicadas de manera presencial.

En cuanto a los documentos revisados, se examinaron los datos correspondientes a la identificación de la organización, capital, número de empleados y descripción de sus productos y servicios, así como el análisis de balances, informes gerenciales y documentos internos, tales como facturas de compra, boletas de honorarios, liquidaciones de sueldos, etc.

Las entrevistas fueron aplicadas a las personas que participan en la gestión y en el proceso de producción de la empresa, como gerente de administración, jefes de planta, operarios, entre otros funcionarios.

La metodología que se utilizó para la aplicación del método de costeo basado en actividades fue la planteada por Kaplan y Cooper (1999). Esta presenta cuatro fases, a saber:

Fase I. Diccionario de actividades, el cual fue construido una vez realizado el diagnóstico de los procesos y los flujos de información.

Fase II. Determinación del gasto de la organización en cada una de las actividades. Este consistió en la identificación de los recursos necesarios para el desarrollo de cada actividad.

Fase III. Identificación de los productos ofrecidos por la empresa.

Fase IV. Selección de los inductores de costos de las actividades que se vinculan con los productos.

La determinación de los inductores se hizo considerando la relación causa efecto y la disponibilidad de información en los sistemas actuales de la empresa. Finalmente, se calcula el costo unitario por metro cúbico para cada uno de los productos fabricados por la empresa y la rentabilidad respectiva.

\section{Resultados}

Fase I. Diccionario de actividades. Las actividades identificadas se especifican en la tabla 1. 
TABLA 1

Diccionario de actividades

\begin{tabular}{|c|c|c|}
\hline \multicolumn{2}{|r|}{ Actividad } & Descripción \\
\hline 1 & Planificar producción & $\begin{array}{l}\text { Planificar los volúmenes de producción de acuerdo con la } \\
\text { demanda esperada. }\end{array}$ \\
\hline 2 & Supervisar proceso productivo & $\begin{array}{l}\text { Supervisar el proceso de extracción y procesamiento de la } \\
\text { materia prima y realizar pruebas técnicas. }\end{array}$ \\
\hline 3 & $\begin{array}{l}\text { Revisar especificaciones y } \\
\text { controlar calidad }\end{array}$ & $\begin{array}{l}\text { Comparar producción con especificaciones técnicas, controlar } \\
\text { calidad de los productos y corregir desviaciones. }\end{array}$ \\
\hline 4 & Extraer y acopiar material & Extraer y acopiar material desde el río o pozos lastreros. \\
\hline 5 & Cargar material extraido & Cargar el material extraído desde el río o pozo al camión. \\
\hline 6 & Transportar material extraido & Transportar el material desde el rio hasta la planta. \\
\hline 7 & Cargar material para venta & Cargar el material al camión para su comercialización. \\
\hline 8 & Cargar material seleccionado & Cargar el material en la malla seleccionadora. \\
\hline 9 & Operar planta procesadora & $\begin{array}{l}\text { Operar y controlar la velocidad de la planta, cargar buzón } \\
\text { alimentador, cargar mallas seleccionadora, entre otras. }\end{array}$ \\
\hline 10 & $\begin{array}{l}\text { Triturar material en chancadora } \\
\text { primario }\end{array}$ & Triturar el material en la chancadora primario. \\
\hline 11 & Trasladar material triturado & Trasladar material triturado por cintas transportadoras. \\
\hline 12 & Triturar material & Triturar el material en la chancadora de cono. \\
\hline 13 & Trasportar material triturado & $\begin{array}{l}\text { Transportar el material disponible para la venta hacia un acopio } \\
\text { de mayor tamaño. }\end{array}$ \\
\hline 14 & $\begin{array}{l}\text { Trasportar y cargar chancadora } \\
\text { impacto }\end{array}$ & Transportar y cargar el material en la chancadora de impacto. \\
\hline 15 & Triturar material forma cúbica & Triturar material para dar forma cúbica. \\
\hline 16 & $\begin{array}{l}\text { Trasportar y cargar material } \\
\text { arenero vibratorio }\end{array}$ & Transportar y cargar el material hacia el arenero vibratorio. \\
\hline 17 & Mantener y reparar máquinas & Realizar reparaciones y mantenciones de las maquinarias. \\
\hline 18 & Transporte productos clientes & Transportar los productos hasta el lugar indicado por el cliente. \\
\hline 19 & Controlar y registrar vehiculos & Controlar $\mathrm{y}$ registrar ingreso $\mathrm{y}$ salida de vehículos $\mathrm{y}$ camiones. \\
\hline 20 & Planificar & Elaborar plan estratégico de la organización \\
\hline 21 & Atender público & Atender potenciales clientes que llegan a la empresa \\
\hline 22 & Realizar cotizaciones de venta & Cotizar a los clientes los productos demandados \\
\hline 23 & Efectuar órdenes de compra & Confeccionar y enviar órdenes de compra a los proveedores \\
\hline 24 & Administrar personal & $\begin{array}{l}\text { Selección, contratación y administración del personal de la } \\
\text { empresa. }\end{array}$ \\
\hline 25 & Realizar pagos a proveedores & Revisar facturas y realizar pagos a los proveedores \\
\hline 26 & $\begin{array}{l}\text { Facturar ventas y cobrar a } \\
\text { clientes }\end{array}$ & Emitir facturas de ventas y realizar cobranza a los elientes \\
\hline 27 & $\begin{array}{l}\text { Evaluar clientes potenciales y } \\
\text { autorizar pagos }\end{array}$ & pagos \\
\hline 28 & $\begin{array}{l}\text { Preparar estados financieros } \\
\text { finales }\end{array}$ & Elaboración de estados financieros finales \\
\hline
\end{tabular}

Fuente: datos de la investigación.

Fase II: Recursos. Corresponde a los costos directos e indirectos incurridos por la empresa en el periodo estudiado. Esta información fue extraída directamente de los documentos e informes del software contable. A continuación se presenta el monto anual de cada recurso (ver tabla 2).

TABLA 2

Recursos y montos asociados (valores expresados en pesos chilenos \$)

\begin{tabular}{|r|l|r|}
\hline \multicolumn{2}{|c|}{ Recurso } & $\begin{array}{c}\text { Monto } \\
\text { (Pesos chilenos \$) }\end{array}$ \\
\hline 1 & Personal administrativo & 66.460 .348 \\
\hline 2 & Personal producción & 326.015 .252 \\
\hline 3 & Equipos y tecnología de producción & 349.537 .454 \\
\hline 4 & Equipos y tecnología de administración & 5.489 .050 \\
\hline 5 & Derechos municipales & 14.482 .398 \\
\hline 6 & Control calidad & 424.000 \\
\hline 7 & Oficina & 28.261 .072 \\
\hline \multicolumn{2}{|c|}{ Total costo de los recursos } & $\mathbf{7 9 0 . 6 6 9 . 5 7 4}$ \\
\hline
\end{tabular}

Fuente: datos de la investigación.

Una vez identificados los recursos, es necesario determinar los factores de distribución o inductores que permitirán la asignación del costo de los recursos a cada una de las actividades identificadas. Para esto fue considerada la relación causa-efecto, entre el recurso y las actividades relacionadas, con lo cual se determinó la fórmula a aplicar a cada recurso (ver tabla 3). 
TABLA 3

Relación recurso, inductor y fórmula de asignación

\begin{tabular}{|c|l|l|l|}
\hline \multicolumn{2}{|c|}{ Recurso } & \multicolumn{1}{c|}{ Inductor } & \multicolumn{1}{c|}{ Fórmula } \\
\hline 1 & $\begin{array}{l}\text { Personal } \\
\text { administrativo }\end{array}$ & $\begin{array}{l}\mathrm{N}^{\circ} \text { personas } \\
\text { administración }\end{array}$ & $\begin{array}{l}\text { \$Personal administrativo / } \\
\text { personas administración }\end{array}$ \\
\hline 2 & Personal producción & $\begin{array}{l}\mathrm{N}^{\circ} \text { personas } \\
\text { producción }\end{array}$ & $\begin{array}{l}\text { \$Personal producción } / \mathrm{N}^{\circ} \\
\text { personas producción }\end{array}$ \\
\hline 3 & $\begin{array}{l}\text { Equipos y tecnología } \\
\text { de producción }\end{array}$ & Horas máquina & $\begin{array}{l}\text { \$ Equipos y tecnologia de } \\
\text { producción / Total horas máquina }\end{array}$ \\
\hline 4 & $\begin{array}{l}\text { Equipos y tecnologia } \\
\text { de administración }\end{array}$ & \% Uso & $\begin{array}{l}\text { \$ Equipos y tecnología de } \\
\text { administración / 100\% }\end{array}$ \\
\hline 5 & $\begin{array}{l}\text { Derechos } \\
\text { municipales }\end{array}$ & $\begin{array}{l}\text { Directo a la } \\
\text { actividad } \mathrm{N}^{\circ} 2\end{array}$ & \$ Directo a la actividad $\mathrm{N}^{\circ} 2$ \\
\hline 6 & Control calidad & $\begin{array}{l}\text { Directo a la } \\
\text { actividad } \mathrm{N}^{\circ} 1\end{array}$ & \$ Directo a la actividad $\mathrm{N}^{\circ} 1$ \\
\hline 7 & Oficina & M2 & \$ Ofícina / M2 totales \\
\hline
\end{tabular}

Fuente: datos de la investigación.

Posteriormente, se procede a calcular el factor de consumo de recursos a cada una de las actividades, utilizando la fórmula expuesta anteriormente, con lo cual se llegó a los resultados que se presentan en la tabla 4.

TABLA 4

Cálculo factor de consumo de recursos (valores expresados en pesos chilenos \$)

\begin{tabular}{|l|r|r|r|r|}
\hline Recurso & $\begin{array}{l}\text { Costo total del } \\
\text { recurso }\end{array}$ & $\begin{array}{c}\text { Uso total } \\
\text { del } \\
\text { inductor }\end{array}$ & Fórmula de cálculo & \multicolumn{1}{|c|}{$\begin{array}{l}\text { Factor de } \\
\text { consumo }\end{array}$} \\
\hline $\begin{array}{l}\text { Personal } \\
\text { administrativo }\end{array}$ & 66.460 .348 & 12 & $66.460 .348 / 12$ & $5.538 .362,33$ \\
\hline Personal producción & 326.015 .252 & 29 & $326.015 .252 / 29$ & $11.241 .905,24$ \\
\hline $\begin{array}{l}\text { Equipos y tecnologia } \\
\text { producción }\end{array}$ & 349.537 .454 & 93.600 & $349.537 .454 / 93.600$ & $3.734,37$ \\
\hline $\begin{array}{l}\text { Equipos y tecnologia } \\
\text { administración }\end{array}$ & 5.489 .050 & $100 \%$ & $5.489 .050 / 100 \%$ & $5.489 .050,00$ \\
\hline $\begin{array}{l}\text { Derechos } \\
\text { municipales }\end{array}$ & 14.482 .398 & 1 & $14.482 .398 / 1$ & $14.482 .398,00$ \\
\hline Control calidad & 424.000 & 1 & $424.000 / 1$ & $424.000,00$ \\
\hline Oficina & 28.261 .072 & 130 & $28.261 .072 / 130$ & $217.392,86$ \\
\hline
\end{tabular}

Fuente: datos de la investigación.

El paso siguiente fue establecer la relación entre el consumo de recursos de cada actividad asignando los costos respectivos, utilizando el factor de consumo calculado en la tabla 4. Esta relación se muestra en la tabla 5, donde es posible observar como el costo de cada recurso fue distribuido entre las actividades que hicieron uso de ese recurso. Como se puede observar el recurso más costoso para la empresa es el equipamiento y tecnología de producción, lo que representa un 44,2\% del costo total de los recursos. Siguen muy de cerca los costos de personal de producción con un $41,2 \%$. En conjunto, estos dos elementos significan un $85,4 \%$ del costo total de la empresa para el periodo estudiado.

Por otro lado, la actividad más costosa es "Operar la planta procesadora" con un 16,5\% del costo total de los recursos, seguida por el "Transporte del material extraído" que significa un 14,1\%, y la "Mantención y reparación de maquinarias" con un 10\%. Ya las actividades menos costosas son "Revisar especificaciones y controlar calidad" con un 0,1\%, y "Planificar, Atender público, Efectuar órdenes de compra y Realizar pagos a proveedores" con un $1 \%$ en cada una de ellas (ver tabla 5 ). 
TABLA 5

Asignación de costo de los recursos a las actividades (valores expresados en pesos chilenos \$)

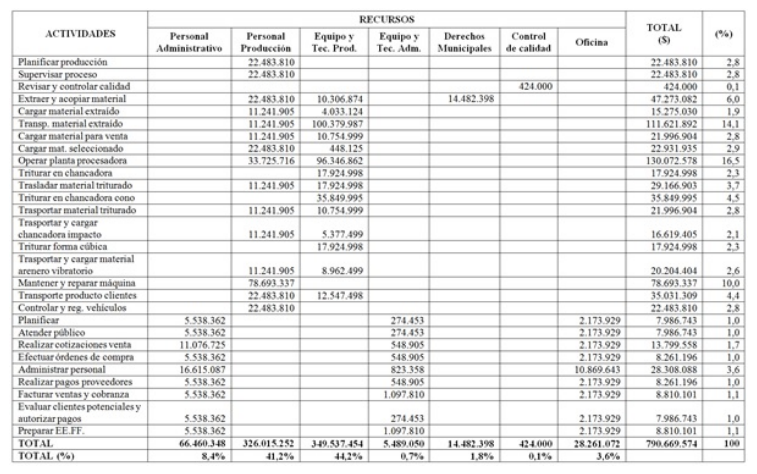

Fuente: datos de la investigación.

Fase III: Objetos de costos. En esta fase se identifican los objetos de costos, es decir, aquellos productos que serán costeados. En la empresa estudiada se producen nueve productos, a saber:

1. Integral de río

2. Bolones

3. Base estabilizada de $1 \frac{1 / 2 "}{}$

4. Grava de $1 \frac{1}{2}$ "

5. Grava de $3 / 4 "$

6. Gravilla de $3 / 4 "$

7. Gravilla cúbica

8. Arena gruesa

9. Arena fina

Fase IV: Inductores de costos para los objetos de costos. En esta fase se determinan los inductores de costos de las actividades al objeto. A partir de ahí, se calcula la tasa de distribución. Dada las características de la empresa, resultó difícil identificar inductores para cada actividad. Sin embargo, fue posible constatar que la principal diferencia entre uno y otro producto radica en la cantidad de actividades necesarias para la producción del cada uno, dado que algunos productos requieren un procesamiento mayor. Por lo mismo, se consideró como principal inductor los metros cúbicos que fueron procesados en cada actividad. Se definieron tres instancias de producción, así:

I. En la primera fase donde una vez extraída la materia prima, es cargada en las bandas seleccionadoras y triturada por la chancadora primaria. En esta primera fase se obtienen el integral de río, los bolones y la base estabilidad de $1 \frac{1}{2} 2$.

II. El resto de material continúa por la malla seleccionadora y se dirige hacia la chancadora de impacto, aquí se obtienen tres nuevos productos, la grava de $1 \frac{1}{2}$ ", grava de $3 / 4$ " y gravilla de $3 / 4$ ".

III. Finalmente, el material que paso por las mallas seleccionadoras en la etapa anterior es dirigido hacia el arenero vibratorio y el triturador cúbico donde se obtienen los últimos tres productos, la gravilla cúbica, arena gruesa y arena fina, según corresponda.

Para las actividades comunes a todos los productos, se consideró como inductor el número de productos, bajo el supuesto de que todos los productos requieren del mismo esfuerzo en dicha actividad. Finalmente, para las actividades relacionadas con las ventas generadas se utilizó como inductor el porcentaje de los ingresos que representa cada producto en particular, estableciendo una relación directa entre el monto facturado y las actividades respectivas. En la tabla 6 se muestran los diferentes inductores y las fórmulas de cálculo para cada una de las actividades. 
TABLA 6

Relación actividad, inductor y fórmula de asignación

\begin{tabular}{|c|c|c|}
\hline Actividad & Inductor & Förmula \\
\hline Planificar producción & Tipos de productos vendidos & S Actividad / Tipos de productos vendidos \\
\hline Supervisar proceso & Tipos de productos vendidos & S Actividad/Tipos de productos vendidos \\
\hline Revisar y controlar calidad & Tipos de productos procesados & $\begin{array}{l}\text { S Actividad / Tipos de productos } \\
\text { procesados }\end{array}$ \\
\hline Extraer y acopiar material & $\begin{array}{l}\text { M3 producidos en primera } \\
\text { instancia }\end{array}$ & $\begin{array}{l}\text { SAtividad / m3 producidos en primera } \\
\text { instancia }\end{array}$ \\
\hline Cargar material extraido & $\begin{array}{l}\text { M3 producidos en primera } \\
\text { instancia }\end{array}$ & $\begin{array}{l}\begin{array}{l}\text { SActividad / m3 producidos en primera } \\
\text { instancia }\end{array} \\
\text { a }\end{array}$ \\
\hline Transportar material extraido & $\begin{array}{l}\begin{array}{l}\text { M3 producidos en primera } \\
\text { instancia }\end{array} \\
\text {. }\end{array}$ & $\begin{array}{l}\text { S Actividad / m3 producidos en primera } \\
\text { instancia }\end{array}$ \\
\hline Cargar material para venta & M3 producidos para la venta & S Actividad / m3 producidos para la venta \\
\hline Cargar material seleccionado & $\begin{array}{l}\text { M3 producidos en segunda } \\
\text { instancia }\end{array}$ & $\begin{array}{l}\text { SActividad / m3 producidos en segunda } \\
\text { instancia }\end{array}$ \\
\hline Operar planta procesadora & M3 procesados en la planta & S Actividad / m3 procesados en la planta \\
\hline Triturar material en chancadora & $\begin{array}{l}\text { M3 producidos en primera } \\
\text { instancia }\end{array}$ & $\begin{array}{l}\text { SActividad/m3 producidos en primera } \\
\text { instancia }\end{array}$ \\
\hline Trasladar material triturado & $\begin{array}{l}\text { M3 producidos en segunda } \\
\text { instancia }\end{array}$ & $\begin{array}{l}\text { SActividad / m3 producidos en segunda } \\
\text { instancia }\end{array}$ \\
\hline Triturar en chancadora cono & $\begin{array}{l}\text { M3 producidos en segunda } \\
\text { instancia }\end{array}$ & $\begin{array}{l}\text { SAtividad / m3 producidos en segunda } \\
\text { instancia }\end{array}$ \\
\hline Trasportar material triturado & $\begin{array}{l}\text { M33 producidos en segunda } \\
\text { instancia }\end{array}$ & $\begin{array}{l}\text { \$A Atetividad / m3 producidos en segunda } \\
\text { instancia }\end{array}$ \\
\hline $\begin{array}{l}\text { Trasportar y cargar chancadora } \\
\text { impacto }\end{array}$ & $\begin{array}{l}\text { M3 producidos en tercera } \\
\text { instancia }\end{array}$ & $\begin{array}{l}\text { SActividad / m3 producidos en tercera } \\
\text { instancia }\end{array}$ \\
\hline Triturar material forma cúbica & M3 gravilla cúbica & S Actividad /m3 gravilla cúbica \\
\hline $\begin{array}{l}\text { Trasportar y cargar material } \\
\text { arenero vibratorio }\end{array}$ & $\begin{array}{l}\text { M3 producidos en tercera } \\
\text { instancia }\end{array}$ & $\begin{array}{l}\text { SActividad / m3 producidos en tercera } \\
\text { instancia }\end{array}$ \\
\hline Mantener y reparar máquina & $\begin{array}{l}\text { M3 producidos en primera } \\
\text { instancia }\end{array}$ & $\begin{array}{l}\text { SActividad / m3 producidos en primera } \\
\text { instancia }\end{array}$ \\
\hline Transporte productos clientes & $\%$ ingresos anuales & S Actividad $/ \%$ ingresos anuales \\
\hline Controlar y reg. vehiculos & Tipos de productos vendidos & S Actividad / Tipos de productos vendidos \\
\hline Planificar & Tipos de productos vendidos & S Actividad/Tipos de productos vendidos \\
\hline Atender público & Tipos de productos vendidos & S Actividad / Tipos de productos vendidos \\
\hline Realizar cotizaciones de venta & $\%$ ing & S Actividad $/ \%$ ingresos anuales \\
\hline Efectuar órdenes de compra & ucidos en primera & $\begin{array}{l}\text { SActividad / m3 producidos en primera } \\
\text { instancia }\end{array}$ \\
\hline Administrar personal & ucidos en primera & dad / m3 producidos en primera \\
\hline Realizar pagos proveedores & $\begin{array}{l}\text { M3 producidos en primera } \\
\text { instancia }\end{array}$ & $\begin{array}{l}\text { \$ \$Actividad / m3 producidos en primera } \\
\text { instancia }\end{array}$ \\
\hline Facturar ventas y cobranza & $\%$ ingresos anuales & S Actividad $/ \%$ ingresos anuales \\
\hline $\begin{array}{l}\text { Evaluar clientes potenciales } y \\
\text { autorizar pagos }\end{array}$ & $\%$ ingresos anuales & S Actividad $/ \%$ ing \\
\hline Preparar EE.FF, & Tipos de productos vendidos & S Actividad / Tipos de productos vendidos \\
\hline
\end{tabular}

Fuente: datos de la investigación.

Posteriormente se calculó la tasa de asignación de cada actividad. Para ello se tomó como base los costos asignados a las actividades en función de los recursos utilizados y se dividió por el uso total del inductor según corresponde. Con esto se obtiene la cuota de costos de actividades que será asignada a cada producto, según el uso que éste haga de la respectividad actividad, y multiplicando la tasa de asignación por la cantidad de inductor utilizada (ver tabla 7 ).

TABLA 7

Cálculo tasa asignación de costo de las actividades a los productos (valores expresados en pesos chilenos \$)

\begin{tabular}{|c|c|c|c|c|}
\hline Actividad & $\begin{array}{l}\text { Costo total } \\
\text { actividad }\end{array}$ & $\begin{array}{l}\text { Inductor } \\
\text { total }\end{array}$ & Fórmula & $\begin{array}{c}\text { Tasa de } \\
\text { asignación }\end{array}$ \\
\hline Planificar producción & 22.483 .810 & 9 & $22.483 .810 / 9$ & $2.498 .201,165$ \\
\hline Supervisar proceso & 22.483 .810 & 9 & $22.483 .810 / 9$ & $2.498 .201,165$ \\
\hline Revisar y controlar calidad & 424.000 & 7 & $424.000 / 7$ & $60.571,4286$ \\
\hline Extraer y acopiar material & 47.273 .082 & 229.726 & $47.273 .082 / 229.726$ & 205,7803 \\
\hline Cargar material extraido & 15.275 .030 & 229.726 & $15.275 .030 / 229.726$ & 66,4924 \\
\hline Transportar material extraido & 111.621 .892 & 229.726 & $111.621 .892 / 229.726$ & 485,8914 \\
\hline Cargar material para venta & 21.996 .904 & 21.131 & $21.996 .904 / 21.131$ & $1.040,9779$ \\
\hline Cargar material seleccionado & 22.931 .935 & 160.749 & $22.931 .935 / 160.749$ & 142,6568 \\
\hline Operar planta procesadora & 130.072 .578 & 208.595 & $130.072 .578 / 208.595$ & 623,5652 \\
\hline Triturar material en chancadora & 17.924.998 & 229.726 & $17.924 .998 / 229.726$ & 78,0277 \\
\hline Trasladar material triturado & 29.166 .903 & 160.749 & $29.166 .903 / 160.749$ & 181,4438 \\
\hline Triturar en chancadora cono & 35.849 .995 & 160.749 & $35.849 .995 / 160.749$ & 223,0185 \\
\hline Trasportar material triturado & 21.996 .904 & 160.749 & $21.996 .904 / 160.749$ & 136,8401 \\
\hline $\begin{array}{l}\text { Trasportar y cargar chancadora } \\
\text { impacto }\end{array}$ & 6.619.405 & 18.459 & $16.619 .405 / 18.459$ & 900,3415 \\
\hline Triturar material forma cúbica & \begin{tabular}{|c|c|c|}
17.924 .998 & \\
\end{tabular} & 60 & $17.924 .998 / 60$ & $98.749,9607$ \\
\hline $\begin{array}{l}\text { Trasportar y cargar material arenero } \\
\text { vibratorio }\end{array}$ & 204.404 & 17.669 & $20.204 .404 / 17.669$ & $1.143,4945$ \\
\hline Mantener y reparar máquina & \begin{tabular}{|c|c|c|c|}
78.337 \\
\end{tabular} & 229.726 & $78.693 .337 / 229.726$ & 342,5530 \\
\hline Transporte productos clientes & 35.031 .309 & $100 \%$ & $35.031 .309 / 100 \%$ & $35.031 .308,83$ \\
\hline Controlar y registrar vehiculos & 22.483 .810 & 9 & & $2.498 .201,165$ \\
\hline Planificar & 7.986 .743 & 9 & $7.986 .743 / 9$ & 887415,9387 \\
\hline Atender público & 7.986 .743 & 9 & $7.986 .743 / 9$ & $887.415,9387$ \\
\hline Realizar cotizaciones de venta & 13.799.558 & $100 \%$ & $13.799 .558 / 100 \%$ & $13.799 .558,28$ \\
\hline Efectuar órdenes de compra & 8.261 .196 & 229.726 & $8.261 .196 / 229.726$ & 35,9611 \\
\hline Administrar personal & 28.308 .088 & 229.726 & $28.308 .088 / 229.726$ & 123,2254 \\
\hline Realizar pagos proveedores & 8.261 .196 & 229.726 & $8.261 .196 / 229.726$ & 35,9611 \\
\hline Facturar ventas y cobranza & 810.101 & $100 \%$ & $8.810 .101 / 100 \%$ & 8.810 .101 \\
\hline $\begin{array}{l}\text { Evaluar clientes potenciales y } \\
\text { autorizar pagos }\end{array}$ & 86.743 & $100 \%$ & $7.986 .743 / 100 \%$ & 7.986 .743 \\
\hline \multirow[t]{2}{*}{ Preparar EE.FF. } & 8.810 .101 & 9 & $8.810 .101 / 9$ & $978.900,1054$ \\
\hline & 790.669 .574 & & & \\
\hline
\end{tabular}

Fuente: datos de la investigación. 
Luego de determinar los inductores de costos de actividades se procede a costear cada producto, obteniendo el costo total por cada uno. Este valor fue dividido por el total de metros cúbicos producidos, con lo cual se obtiene el costo unitario. Como se puede observar el producto que recibió la mayor parte de los costos fue el $\mathrm{N}^{\circ} 3$ (Base Estabilizada) con un 52,7\% de los costos totales. Éste resulta ser también el producto que más se produce, con un 59,8\% del volumen total producido. Por su parte, el producto que recibió la menor parte de los costos fue el $\mathrm{N}^{\circ} 2$ (Bolones) con un 2,3\% de estos. Sin embargo, este producto no es el que menos se produce, sino el que menos procesamiento requiere, dado que se vende sin transformación.

Por otra parte, el Producto $\mathrm{N}^{\circ} 7$ (Gravilla Cúbica) es el que tiene un menor volumen de producción, con tan solo un $0,03 \%$ de ésta. Sin embargo, el porcentaje de costos asignados fue del 3,6\%, dado que para su fabricación se requiere de la realización de actividades específicas como son el procesamiento en la chancadora de impacto, la cual disminuye el calibre de la gravilla de $3 / 4 \mathrm{y}$ le da la forma cúbica. Este proceso significa un costo alto dado el volumen procesado (ver tablas 8 y 9 ).

TABLA 8

Asignación del costo de las actividades a los productos (valores expresados en pesos chilenos \$)

\begin{tabular}{|c|c|c|c|c|c|}
\hline \multirow[b]{2}{*}{ ACTIVIDAD } & \multicolumn{5}{|c|}{ OBJETO DE $\cos T O S$} \\
\hline & $\begin{array}{l}\text { Integral de } \\
\text { Rio }\end{array}$ & Bolones & $\begin{array}{c}\text { Base } \\
\text { Estabilizada }\end{array}$ & $\begin{array}{l}\text { Grava de } \\
11 / 2, "\end{array}$ & $\begin{array}{l}\text { Grava de } \\
\text { y/y de }\end{array}$ \\
\hline Planificar producción & 2.498 .201 & 2.498 .201 & 2.498.201 & 2.498 .201 & 2.498 .201 \\
\hline Supervisar proceso & 2.498 .201 & 2.498 .201 & 2.498 .201 & 2.498 .201 & 2.498 .201 \\
\hline Revisar y controlar calidad & & & 60.571 & 60.571 & 60.571 \\
\hline Extraer y acopiar material & 3.760 .429 & 587.914 & 28.282 .031 & 5.045 .733 & 2.162 .545 \\
\hline Cargar material extraido & 1.215 .082 & 189.969 & 9.138 .580 & 1.630 .393 & 698.768 \\
\hline Transportar material extraido & 8.879 .180 & 1.388.192 & 66.779 .945 & 11.914 .058 & 5.106 .233 \\
\hline Cargar material para venta & 19.022 .830 & 2.974.074 & & & \\
\hline Cargar material seleccionado & & & 19.606 .463 & & \\
\hline Operar planta procesadora & & & 85.701 .551 & 15.289 .818 & 6.553 .046 \\
\hline Triturar material en chancadora & 1.425 .879 & 222.925 & 10.723 .975 & 1.913 .240 & 819.993 \\
\hline Trasladar material triturado & & & 24.937 .267 & & \\
\hline Triturar en chancadora cono & & & 30.651 .212 & & \\
\hline Trasportar material triturado & & & 18.807 .025 & & \\
\hline \multicolumn{6}{|l|}{$\begin{array}{l}\text { Trasportar y cargar chancadora } \\
\text { impacto }\end{array}$} \\
\hline \multirow{2}{*}{\multicolumn{6}{|c|}{$\begin{array}{l}\text { Triturar material forma cúbica } \\
\text { Trasportar y cargar material }\end{array}$}} \\
\hline & & & & & \\
\hline Mantener y reparar máquina & 6.259 .814 & 978.674 & 47.079 .803 & 8.399 .400 & 3.599 .890 \\
\hline Transporte productos clientes & 832.372 & 509.939 & 20.025 .301 & 3.661 .985 & 2.411 .649 \\
\hline Controlar y registrar vehículos & 2.498 .201 & 2.498 .201 & 2.498 .201 & 2.498 .201 & 2.498 .201 \\
\hline Planificar & 887.416 & 887.416 & 887.416 & 887.416 & 887.416 \\
\hline Atender público & 887.416 & 887.416 & 887.416 & 887.416 & 887.416 \\
\hline Realizar cotizaciones de venta & 327.889 & 200.876 & 7.888 .381 & 1.442 .532 & 949.999 \\
\hline Efectuar órdenes de compra & 657.153 & 102.741 & 4.942 .419 & 881.766 & 377.915 \\
\hline Administrar personal & 2.251 .822 & 352.055 & 16.935.858 & 3.021 .488 & 1.294 .976 \\
\hline Realizar pagos proveedores & 657.153 & 102.741 & 4.942 .419 & 881.766 & 377.915 \\
\hline Facturar ventas y cobranza & 209.335 & 128.246 & 5.036 .207 & 920.961 & 606.511 \\
\hline $\begin{array}{l}\text { Evaluar clientes potenciales y } \\
\text { autorizar pagos }\end{array}$ & 189.771 & 116.260 & 4.565 .543 & 834.891 & 549.829 \\
\hline Preparar EE.FF. & 978.900 & 978.900 & 978.900 & 978.900 & 978.900 \\
\hline \multirow[t]{2}{*}{ COSTO TOTAL (\$) } & 55.937 .043 & 18.102.941 & 416.352 .888 & 66.146.937 & 35.818 .177 \\
\hline & $7,1 \%$ & $2,3 \%$ & $52,7 \%$ & $8,4^{\circ} \%$ & $4,5 \%$ \\
\hline COSTO UNITARIO & 3.061 & 6.336 & 3.029 & 2.698 & 3.408 \\
\hline
\end{tabular}

Fuente: datos de la investigación. 
TABLA 9

Asignación del costo de las actividades a los productos (valores expresados en pesos chilenos \$)

\begin{tabular}{|c|c|c|c|c|c|}
\hline \multirow{2}{*}{ ACTIVIDAD } & \multicolumn{5}{|c|}{ OBJETO DE COSTOS } \\
\hline & Gravilla "\%" & Gravilla cubica & $\begin{array}{l}\text { Arena } \\
\text { gruesa }\end{array}$ & Arena fina & Total (\$) \\
\hline Planificar producción & 2.498 .201 & 2.498 .201 & 2.498 .201 & 2.498 .201 & 22.483 .810 \\
\hline Supervisar proceso & 2.498 .201 & 2.498 .201 & 2.498 .201 & 2.498 .201 & 22.483 .810 \\
\hline Revisar y controlar calidad & 60.571 & 60.571 & 60.571 & 60.571 & 424.000 \\
\hline Extraer y acopiar material & 3.786 .151 & 12.347 & 2.637 .486 & 998.446 & 47.273 .082 \\
\hline Cargar material extraido & 1.223 .393 & 3.990 & 852.233 & 322.621 & 15.275 .030 \\
\hline Transpotar material extraido & 8.939 .916 & 29.153 & 6.227 .670 & 2.357 .545 & 111.621 .892 \\
\hline $\begin{array}{l}\text { Cargar material } \\
\text { Crenai }\end{array}$ & & & & & 21.996 .904 \\
\hline Cargar material & 2.624 .742 & 8.559 & & 692.171 & 22.931 .935 \\
\hline Operar planta procesadora & 11.472 .976 & 37.414 & 7.992 .235 & 3.025 .538 & 130.072 .578 \\
\hline $\begin{array}{l}\text { Triturar material en } \\
\text { chancadora }\end{array}$ & 1.435 .632 & 4.682 & 1.000 .081 & 378.591 & 17.924 .998 \\
\hline Trasladar material triturado & 3.338 .384 & 10.887 & & 880.365 & 29.166 .903 \\
\hline Triturar en chancadora cono & 4.103 .317 & 13.381 & & 1.082 .086 & 35.849 .995 \\
\hline Trasportar material triturado & 2.517 .720 & 8.210 & & 663.948 & 21.996 .904 \\
\hline $\begin{array}{l}\text { Trasportar y cargar chancadora } \\
\text { impacto }\end{array}$ & 16.565 .384 & 54.020 & & & 16.619 .405 \\
\hline Triturar material forma cúbica & & 17.924 .998 & & & 17.924 .998 \\
\hline $\begin{array}{l}\text { Trasportar y cargar material } \\
\text { arenero vibratorio }\end{array}$ & & & 14.656 .169 & 5.548 .235 & 20.204 .404 \\
\hline Mantener y reparar máquina & 6.302 .633 & 20.553 & 4.390 .502 & 1.662 .067 & 78.693.337 \\
\hline Transporte productos clientes & 4.222 .280 & 19.670 & 2.287 .677 & 1.060 .436 & 35.031 .309 \\
\hline Controlar y registrar vehiculos & 2.498 .201 & 2.498 .201 & 2.498 .201 & 2.498 .201 & 22.483 .810 \\
\hline Planificar & 887.416 & 887.416 & 887.416 & 887.416 & 7.986 .743 \\
\hline Atender público & 887.416 & 887.416 & 887.416 & 887.416 & 7.986 .743 \\
\hline Realizar cotizaciones de venta & 1.663 .243 & 7.748 & 901.163 & 417.728 & 13.799 .558 \\
\hline Efectuar órdenes de compra & 661.648 & 2.158 & 460.913 & 174.483 & 8.261 .196 \\
\hline Administrar personal & 2.267 .225 & 7.394 & 1.579 .380 & 597.890 & 28.308 .088 \\
\hline Realizar pagos proveedores & 661.648 & 2.158 & 460.913 & 174.483 & 8.261 .196 \\
\hline Facturar ventas $y$ cobranza & 1.061 .870 & 4.947 & 575.333 & 266.691 & 8.810 .101 \\
\hline $\begin{array}{l}\text { Evaluar clientes potenciales y } \\
\text { autorizar pagos }\end{array}$ & 96 & 35 & 565 & 241.767 & 7.986 .743 \\
\hline Preparar EE.FF. & 978.900 & 978.900 & 978.900 & 978.900 & 8.810 .101 \\
\hline \multirow[t]{2}{*}{ COSTO TOTAL (\$) } & 84.119 .703 & 28.485 .660 & 54.852 .228 & 30.853 .998 & 790.669 .574 \\
\hline & $10,6 \%$ & $3,6 \%$ & $6,9 \%$ & $3,9 \%$ & $100 \%$ \\
\hline COSTO UNITARIO & 4.572 & 474.761 & 4.280 & 6.359 & \\
\hline
\end{tabular}

Fuente: datos de la investigación.

$\mathrm{Al}$ revisar los costos unitarios es posible observar que el producto más costoso es la gravilla cúbica, dado su bajo volumen de producción (solo $60 \mathrm{~m} 3$ ). Con esto se obtiene un costo por metro cúbico de $\$ 474.761$, que, frente al precio de venta actual de $\$ 9.000$, implica un margen negativo de (465.761), lo que representa un $(5.175 .1 \%)$ (ver tabla 10$)$.

\section{TABLA 10}

Utilidad por metro cúbico por tipo de producto (valores expresados en pesos chilenos \$)

\begin{tabular}{|c|c|c|c|c|c|c|c|c|c|}
\hline & $\begin{array}{l}\text { Integral } \\
\text { de Rio }\end{array}$ & Bolones & $\begin{array}{c}\text { Base } \\
\text { Estab. }\end{array}$ & $\begin{array}{c}\text { Grava } 1 \\
1 / 2,1\end{array}$ & ava & $\begin{array}{l}\text { Gravill } \\
a^{3} \% "\end{array}$ & $\begin{array}{l}\text { Gravilla } \\
\text { cúbica }\end{array}$ & $\begin{array}{l}\text { Arena } \\
\text { gruesa }\end{array}$ & $\begin{array}{c}\text { Arena } \\
\text { fina }\end{array}$ \\
\hline & 3.000 & 4.900 & 4.000 & 4.100 & 6.300 & 6.300 & 9.000 & 4.900 & 6.000 \\
\hline & 3.061 & 6.336 & 3.029 & 2.698 & 3.408 & 4.572 & 474.761 & 4.280 & $6.35 \mathrm{~s}$ \\
\hline Utilidad & & $(1.436)$ & 971 & & 892 & & $(465.761)$ & 620 & (359) \\
\hline$\%$ utilidad & $2,0 \%$ & $29.3 \%$ & $24.3 \%$ & $34,2 \%$ & $45,9 \%$ & $27,4 \%$ & $5.175,1 \%$ & $12,7 \%$ & $6,0 \%$ \\
\hline
\end{tabular}

Fuente: datos de la investigación.

Ahora bien, el producto más rentable resulta ser la Gravilla 3/4", con una ganancia del 45,9\%, y una producción de solo un $8,01 \%$ del total producido por la empresa. Esto implica apenas un $10,6 \%$ de los costos totales de producción. Este resultado puede mostrar una oportunidad para la empresa, en la medida que tenga demanda suficiente para aumentar los volúmenes de producción de este producto en particular, y busque alternativas para su producción.

\section{Discusión y conclusiones}

Una vez aplicado el método de costeo basado en actividades es posible concluir que, para identificar de forma más precisa las actividades, es necesario conocer los procesos dentro de la empresa. Esto concuerda con Brito et al. (2009), quienes señalan que el sistema ABC se debe centrar en las actividades que realiza la 
empresa siguiendo el flujo de los procesos. Para esto, es imprescindible que la empresa cuente con manuales de procedimientos que ayuden a la comprensión de los procesos y tareas desarrolladas por la empresa.

En lo que respecta a la obtención del costo de las actividades, es importante primero identificar los recursos utilizados para la realización de las mismas. El costo de estos recursos puede ser obtenido directamente desde la contabilidad financiera de la empresa, donde se registran los desembolsos y ajustes contables de la organización. La fase más crítica al implementar el $\mathrm{ABC}$ es la determinación de los inductores. Es fundamental tener un buen conocimiento de la empresa para lograrlo, especialmente de su proceso productivo. Por lo anterior, se requiere que esta labor sea realizada por un equipo multidisciplinario, de cara a aminorar los errores que pudieran presentarse.

En este contexto, el sistema de información es clave, pues se debe mantener un control de los tiempos reales de horas hombre, horas máquinas, metros cúbicos procesados, entre otros registros necesarios para realizar cada actividad. Igualmente, se debe controlar cada producto u objeto de costo, con el objetivo de que la aplicación de los inductores sea más precisa y por ende se obtenga un costo más exacto. Sin perjuicio de lo anterior, en una pequeña empresa hay que evitar el excesivo número de inductores, puesto que el trabajo que implica mantener el sistema de información puede encarecer la aplicación del modelo, corriendo el riesgo de su abandono. Ahora bien, aun cuando se utilicen un número reducido de inductores y que estos sean más bien de transacción y de duración, es recomendable hacer una mejor distribución de los costos. Con esto se logra determinar cuáles actividades son ejecutadas solo con el fin de producir determinados productos. Por lo tanto, dichos costos deberían ser asignados a esos productos en específico.

En cuanto a las oportunidades de reducción de costos identificadas, se evidencia que una de las actividades más costosas para la empresa es la de trasportar el material del río hacia la planta. Dado lo anterior, se propone a la empresa revisar la necesidad de este traslado y la forma de operar del mismo. Esto con el fin de identificar oportunidades de reducción, como por ejemplo la disminución del número de traslados diarios, de la distancia recorrida, del cambio de tecnología, o de la externalización del servicio. Asimismo, se sugiere revisar los costos relacionados con la mantención y reparación de maquinarias, identificando el origen de estas reparaciones para preveer su posible disminución.

Con lo anterior, queda demostrado que el $\mathrm{ABC}$ no sólo es útil para la determinación del costo final de los productos o servicios, como método de asignación de costos indirectos, sino también para identificar aquellas actividades más costosas y evaluar alternativas para la reducción de sus costos.

En lo que respecta a las decisiones sobre productos, se sugiere aumentar el volumen de gravilla $3 / 4$ " cuando los niveles de demanda así lo permitan y exista mercado para ello. En cuanto a la gravilla cúbica, a pesar de que el volumen vendido es bajo, no es posible eliminarlo, dado que los clientes realizan la compra de todos los productos que requieren para su trabajo. Al no contar con este insumo, estos realizarían el pedido con otro proveedor, con el fin de realizar un solo despacho. Por lo anterior, se sugiere hacer alianzas estratégicas con competidores o posibles proveedores de gravilla cúbica, para que una sola empresa se encargue de la producción de este material y haga la distribución a todos los oferentes.

Finalmente, es preciso señalar que la principal limitación presentada en este estudio fue la falta de información para la determinación de inductores asociados al tiempo. Esto puede afectar la precisión del costo obtenido. Sin embargo, el diseño de este sistema y la metodología propuesta, permitiría, una vez implementado un buen sistema de información, llegar a un costo más preciso en el futuro.

\section{Referencias}

Arellano, O., Quispe, G., Ayaviri, D., \& Escobar, F. (2017). Estudio de la aplicación del método de Costos ABC en las Mypes del Ecuador. Revista de Investigaciones Altoandinas, 19(1), 33-46. https://dx.doi.org/10.18271/ria.2 016.253 
Artieda, C. (2015). Análisis de los sistemas de costos como herramientas estratégicas de gestión en las pequeñas y medianas empresas (PYMES). Revista publicando, 2(2), 90-113. https://dialnet.unirioja.es/servlet/articulo?co digo $=5833491$

Baldini, R., \& Casari, M. (2008). Los modelos de costeo y la gestión empresaria. Invenio, 11(20), 73-89. DialnetLosModelosDeCosteoYLaGestionEmpresariaSegundaParte-4257125.pdf

Balmaceda, M., \& Guerra, L. (2017). Metodologías de costeo de educación superior (Seminario para optar al título de Ingeniero Comercial, Mención Administración). Recuperado de http://repositorio.uchile.cl/bitstream/handle /2250/143442/Seminario\%20de\%20Titulo.pdf?sequence=1yisAllowed $=\mathrm{y}$

Bhimani, A., Gosselin, M., Ncube, M., \& Okano, H. (2007). Activity-Based Costing: How far have we come internationally? Cost Management, 21(3), 12-17. http://eprints.lse.ac.uk/6033/

Brito, J., Ferreiro, V., \& López, C. (2009). Sistema de costos basado en actividades en la fabricación de campanas industriales: aplicación del modelo $\mathrm{ABC}$ como herramienta de gestión. XV Congreso Internacional de Contaduría, Administración e Informática. Asociación Nacional de Facultades y Escuelas de Contaduría y Administración. México.

Bustamante, A. (2015). Costeo basado en actividades. Revisión de la literatura. Revista CEA, 1(1), 109-119. https:// doi.org/https://doi.org/10.22430/

Cárdenas, S. (2011). Una aproximación al uso de herramientas de gerencia estratégica de costos en instituciones privadas de educación superior. Cuadernos de Contabilidad, 12(31), 547-569. http://www.scielo.org.co/scielo.p hp?script=sci_arttext\&pid=S0123-14722011000200007

Cataldo, P. (2008). Modelamiento de un sistema de costeo basado en actividades para el proceso de flotación de la compañía minera doña Inés de Collahuasi. Tesis de Maestría. Universidad de Chile, Santiago, Chile.

Chea, A. (2011). Activity-Based costing system in the service sector: A strategic approach for enhancing managerial decision making and competitiveness. International Journal of Business and Management, 6(11), 3-10. https:// doi.org/10.5539/ijbm.v6n11p3

Coronel, C. (2014). Aplicacion del sistema de costos por actividades y su efecto en la rentabilidad de la empresa Cementos Selva S.A. Tesis para obtar al título de Contador Público). Universidad de San Martín, Tarapoto, San Martín, Perú.

Cuevas, C., Chávez, G., Castillo, J., Marino, N., \& Solarte, W. (2004). Costeo ABC ¿Por qué y cómo implantarlo? Estudios gerenciales, 20(92), 47-103. http://www.scielo.org.co/scielo.php?script=sci_arttext\&pid=S0123-5923 2004000300003

Fei, Z. Y., \& Isa, C. R. (2010). Factors influencing activity-based costing success: A research framework. International Journal of Trade, Economics and Finance, 1(2), 144-150. http://www.ijtef.org/papers/26-C130.pdf

Gómez, M. (2014). Diseño de un sistema ABC para las empresas comercializadoras y distribuidoras de productos perecederos. Tesis de Maestría. Universidad de los Andes, Venezuela.

Kaplan, R. S., \& Cooper, R. (2003). Coste y Efecto: cómo usar el ABC, el ABM y el ABB para mejorar la gestión, los procesos y la Rentabilidad. Barcelona: Editorial Gestión.

Lima, L., \& Júnior, W. (2017). Custeio Baseado em Atividades (ABC) no setor de compra e venda de café em uma empresa de grande porte. Custos e agronegócio on line, 13(1), 206-238. http://www.custoseagronegocioonline.c om.br/numero1v13/OK\%209\%20ABC.pdf

López, M., Gómez, A., \& Marín, S. (2011). Sistema de costos ABC en la mediana empresa industrial mexicana. Cuadernos de contabilidad, 12(30), 23-43. http://www.scielo.org.co/scielo.php?script=sci_arttext\&pid=S0123 $-14722011000100002$

Martín F. (2017). Contabilidad de costes. Madrid: Ediciones académicas S.A.

Martins, E. (2008). Contabilidade de custos, $9^{a}$ ed. São Paulo: Editorial Atlas.

Medianeira Stefano, N. (2011). Gerenciamento de custos em pequenas empresas prestadoras de serviço utilizando o activity based costing (ABC). Estudios Gerenciales, 27(121), 15-38. https://doi.org/10.1016/S0123-5923(11) 70179-6 
Molina, V., \& Reyes, M. (2011). Diseño de un sistema de contabilidad de costos para la empresa agrícola Agrofepp ubicada en el sector de Rafael periodo primer trimestre del 2009. Tesis de Grado. presentada previo a la obtención del Título de Ingeniería en Contabilidad y Auditoría CPA). Universidad Técnica de Cotopaxi, Latacunga, Ecuador.

Morillo, M. \& Cardozo, C. (2017). Sistema de costos basado en actividades en hoteles cuatro estrellas del estado Mérida, Venezuela. Innovar, 27(64), 91-114. https://doi.org/10.15446/innovar.v27n64.62371.

Prieto, M., Santidrián, A., \& Valladares, H. (2007). El sistema ABC en el sector logístico mexicano: un análisis empírico. Revista Iberoamericana de Contabilidad de Gestión, 10, 13-56. http://www.observatorio-iberoamericano.org/ri cg/N\%C2\%BA_10/Bego\%C3\%B1a_Prieto_y_otros.pdf

Prieto, M., \& Valladares, H. (2013). Beneficios y limitaciones del modelo ABC: análisis de un caso del sector logístico mexicano. Cofin Habana, 1, 18-30. https://www.academia.edu/21351625/Beneficios_y_limitaciones_del_modelo_ABC_an\%C3\%A1lisis _de_un_caso_del_sector_log\%C3\%ADstico_mexicano_ABC_model_s_benefits_and_limitations_analysis_o f_a_Mexican_logistic_sector_case

Raupp, F., \& Beuren, I. (2011). Metodologias de custos utilizadas nas maiores indústrias do setor têxtil do Estado de Santa Catarina. Revista Ciências Administrativas, 17(3), 973-1000. https://periodicos.unifor.br/rca/article/vie w/3299

Reinheimer, C., González, B., \& Zanitti, L. (2010). Sistema de costeo basado en actividades - Implementación del método $A B C$ en una Pyme como herramienta de gestión. Universidad Tecnológica Nacional Facultad Regional Santa Fe. Recuperado de http://www.edutecne.utn.edu.ar/PPI-CAI/ppi2004.pdf

Ríos, M., Rodríguez, M., \& Ferrer, J. (2012). Los costos basados en actividades como herramienta de gestión en las Pymes. El caso de las empresas de servicios en México. Revista Iberoamericana de Contabilidad de Gestión, 10(19), 1-21. http://www.observatorio-iberoamericano.org/RICG/N\%C2\%BA_19/Martha_Rios;_M\%C2\% AA_Lourdes_Rodriguez-Vilari\%C3\%B1o_y_Juli\%C3\%A1n_Ferrer.pdf

Ríos, M., Muñoz, C., \& Rodríguez, L. (2014). Is the activity based costing system a viable instrument for small and medium enterprises? The case of Mexico. Estudios Gerenciales, 30(132), 220-232. https://doi.org/10.1016/j.es tger.2014.02.014

Sánchez, B. (2013). Implicancias del método de Costeo ABC. Quipukamayoc. Revista de la Facultad de Ciencias Contables, 21(39), 65-73. https://doi.org/10.15381/quipu.v21i39.6273.

Sanhueza, M. (2015). Diseño de un Modelo de Costeo ABC para la reparación de buques atuneros, Tesis. Universidad del Bío-Bío, Chile.

Santos, J., \& Padrones, A. (2015). Método de Custeio Baseado em Atividades para Educação Básica: uma proposta de aplicação. XII Congresso Brasileiro de Custos. Asociación Brasileña de Costos, Foz do Iguaçu, Brasil.

Rincón, C. (2011). Presupuestos empresariales, 1ª ed. Bogotá: Ecoe ediciones.

Strasorier, M. (2012). Diseño de un sistema de costos para una empresa dedicada a la extracción y comercialización de triturados pétreos. Tesis para obtar al título de Contador Público. Universidad Empresarial Siglo 21, Córdoba, Argentina.

Toro, F. (2016). Costos ABC y presupuestos. Costeo con base en actividades productivas. Bogotá: Ecoe Ediciones.

Torres, C., Salete, M., \& Delgado, C. (2017). Costeo de productos en la industria panadera utilizando el método ABC. Revista Interciencia, 42(10), 646-652. https://www.interciencia.net/wp-content/uploads/2017/10/646-TOR RES-42_107.pdf

Vieira, E., Sausen, J., Schreiber, R., \& Dalla Nora, L. (2016). Custeio baseado em atividades da retaguarda operacional de uma instituição financeira. XXIII Congresso Brasileiro de Custos. Asociación Brasileña de Costos, Porto de Galinhas, Brasil.

\section{Notas}

* Artículo de investigación científica y tecnológica.

1 También pueden ser llamados "inductor de costos" o "generador de costos". 
Cecilia del Pilar Gallegos Muñoz, et al. Gestión de costos en el sector de áridos a través del mét...

Licencia Creative Commons CC BY 4.0

Para citar este articulo: Gallegos M., C P., \& Rodríguez Q., E. I. (2020). Gestión de costos en el sector de áridos a través del método de costeo basado en actividades. Cuadernos de Contabilidad, 21. https://doi.org /10.11144/Javeriana.cc21.gcsa 